\title{
Neurodegeneration: the first mechanistic therapy and other progress in 2017
}

\author{
John Hardy, \\ Reta Lila Weston Research Laboratories and Department of Molecular \\ Neuroscience, \\ UCL Institute of Neurology, \\ London \\ WC1N 3BG \\ UK
}

j.hardy@ucl.ac.uk

While the news about mechanistic therapies in neurodegeneration has been generally rather glum, particularly with the failure of BACE trials for Alzheimer's disease, in my view, easily the most important development in neurodegeneration research in 2017 has been introduction of successful gene based therapies for spinal muscular atrophy which is caused by mutations in the SMN1 gene $(1,2)$. One report, a phase $1 / 2$ trial, depends on injection of adenoassociated virus carrying SMN cDNA (1) and the other, a report of a phase 3 trial, depends upon intrathecal injection of an antisense oligonucleotide which corrects the misplicing of the SMN2 pseudogene such that this pseudogene produces enough active protein for the defect to be corrected (2). The phase 3 trial of the antisense oligonucleotide was successful to the extent that the trial was halted early. Remarkably, this therapy is now in clinical practice. This is clearly important for patients with SMA though discussions over the price of the therapy have somewhat soured the elation. Its wider importance however, is that it is the first mechanistic therapy for a neurodegenerative disease and, while this therapeutic approach for SMA uniquely depends on the activation of a pseudogene, it bodes well for oligonucleotide strategies for other genetic diseases: APP for Alzheimer's disease, MAPT for frontotemporal dementia and progressive supranuclear palsy, synuclein for Parkinson's disease, huntingtin for Huntington's disease and so on. Let us hope that this breakthrough is the first swallow of a treatment spring for all these devastating diseases. 


\section{7: Neurodegeneration Progress}

The Nobel Prize for Chemistry this year went to Jacques Dubochet, Joachim Frank and Richard Henderson for their work developing the cryo-e.m. technology. This year, the power of this approach has been demonstrated by the identification of structures for the structure of the paired helical filaments of tangle (3) and for amyloid fibrils of plaques (4). In both cases, we already know that there are multiple different structures which are associated with different clinical features (3-5).

For many years, we have lacked biomarkers which are effective and easily accessible markers of neurodegeneration. PET and CSF markers can be useful of course, but a blood biomarker of neurodegeneration would have the potential to transform the field. Neurofilament light protein (NFL) in blood seems to be such a marker. It seems likely that its level increases in blood in all diseases where there is ongoing axonal damage. The use of this marker has been reported in Huntington's disease and in Alzheimer's disease $(6,7)$ but there are other reports submitted or "in press" which indicate this is a general marker of damage. This marker offers the hope of being able to measure changes in the rate of neurodegeneration and of assessing treatment effects in a systematic, inexpensive and consistent fashion. It, therefore, has the potential to revolutionise clinical trials.

Perhaps the biggest advance in genetics this year has been from the release of the data from the GTEx project (8). This online resource maps gene expression with genotype across all the major tissues and will be a Rosetta Stone for the interpretation of genome wide association studies. As single nucleotide polymorphisms are associated with disease, these can be dialed up to see which gene, which transcript and which tissue they alter expression in. The importance of data integration between genetic and expression data was beautifully illustrated by the work showing that many of the Alzheimer loci were targets of the microglial transcription factor PU.1 (9)... extending earlier studies which increasingly show the importance of the microglial response in Alzheimer's disease.

Besides the progress in developing biomarkers and understanding the genetic bases of neurodegeneration, there has also been an enormous amount of work 
showing that pathology templating is an important issue. There has been work showing $A \beta$, tau and synuclein pathology can template... in this area it is difficult to choose a single paper, but work in this area was initiated by Jucker and Walker so their recent review (10) is probably the place to start. Quite how the genetic findings in these diseases fit with the clear evidence of pathology propagation remains unclear because, as yet, there is no clear link between the two sets of investigations.

My favourite paper of the year, I have to say, is a little off the wall and not directly related to neurodegeneration. Because of my interest in ALS I had set a PubMed search on RNA splicing. Through this search I pulled up a finding in cephalopods. Octopuses and squid are famously intelligent and genetically complex organisms and like us, generate much diversity from a limited number of genes. We, and all mammals, generate this gene diversity through alternate splicing (though a very few genes such as a 5HT receptor and a glutamate receptor do have consequential and variable RNA editing). Octopuses and squid however, generate most of their protein diversity by RNA editing.... This unexpected finding shows there is an enormous amount left to discover in all areas of science, including neurodegeneration: life truly is amazing; worth considering as you crunch your next plate of calamari (11).

\section{References}

1) Mendell JR, Al-Zaidy S, Shell R, et al. Single-dose gene-replacement therapy for spinal muscular atrophy. N Engl J Med 2017; 377: 1713-22.

2) Finkel RS, Mercuri E, Darras BT, et al. Nusinersen versus sham control in infantile-onset spinal muscular atrophy. N Engl J Med 2017; 377: 1723-32.

3) Fitzpatrick AWP, Falcon B, He S, Murzin AG, Murshudov G, Garringer HJ, Crowther RA, Ghetti B, Goedert M, Scheres SHW. Cryo-EM structures of tau filaments from Alzheimer's disease. Nature. 2017 Jul 13;547(7662):185-190.

4) Gremer L, Schölzel D, Schenk C, Reinartz E, Labahn J, Ravelli RBG, Tusche M, Lopez-Iglesias C, Hoyer W, Heise H, Willbold D, Schröder GF. Fibril 
structure of amyloid- $\beta(1-42)$ by cryo-electron microscopy. Science. 2017 Oct 6;358(6359):116-119.

5) Qiang W, Yau WM, Lu JX, Collinge J, Tycko R. Structural variation in amyloid- $\beta$ fibrils from Alzheimer's disease clinical subtypes. Nature. 2017 Jan 12;541(7636):217-221.

6) Byrne LM, Rodrigues FB, Blennow K, Durr A, Leavitt BR, Roos RAC, Scahill RI, Tabrizi SJ, Zetterberg H, Langbehn D, Wild EJ. Neurofilament light protein in blood as a potential biomarker of neurodegeneration in Huntington's disease: a retrospective cohort analysis. Lancet Neurol. 2017 Aug;16(8):601-609.

7) Weston PSJ, Poole T, Ryan NS, Nair A, Liang Y, Macpherson K, Druyeh R, Malone IB, Ahsan RL, Pemberton H, Klimova J, Mead S, Blennow K, Rossor MN, Schott JM, Zetterberg H, Fox NC. Serum neurofilament light in familial Alzheimer disease: A marker of early neurodegeneration. Neurology. 2017 Oct 25. pii: 10.1212/WNL.0000000000004667. doi: 10.1212/WNL.0000000000004667. [Epub ahead of print]

8) GTEx Consortium. Genetic effects on gene expression across human tissues. Nature. 2017 Oct 11;550(7675):204-213.

9) Huang KL, Marcora E, Pimenova AA, et al. A common haplotype lowers PU.1 expression in myeloid cells and delays onset of Alzheimer's disease. Nat Neurosci. 2017 Aug;20(8):1052-1061.

10)Rasmussen J, Jucker M, Walker LC. A $\beta$ seeds and prions: How close the fit? Prion. 2017 Jul 4;11(4):215-225.

11)Liscovitch-Brauer N, Alon S, Porath HT, Elstein B, Unger R, Ziv T, Admon A, Levanon EY, Rosenthal JJ, Eisenberg E. Trade-off between transcriptome plasticity and genome evolution in cephalopods. Cell. 2017 Apr 6;169(2):191-202 